\title{
Diagnóstico e Tratamento da COVID-19: protocolo de scoping review
}

\author{
Diagnosis and Treatment of COVID-19: scoping review protocol \\ Diagnóstic y Tratamiento de COVID-19: protocolo de revisión de alcance
}

Recebido: 19/03/2021 | Revisado: 26/03/2021 | Aceito: 30/03/2021 | Publicado: 10/04/2021

\author{
Carina Bortolato-Major \\ ORCID: https://orcid.org/0000-0002-7711-8010 \\ Universidade Estadual do Norte do Paraná, Brasil \\ E-mail: cabortolato@uenp.edu.br \\ Aline Balandis Costa \\ ORCID: https://orcid.org/0000-0003-4339-6204 \\ Universidade Estadual do Norte do Paraná, Brasil \\ E-mail: alinebalandis@uenp.edu.br \\ Camila Dalcol \\ ORCID: https://orcid.org/0000-0002-6224-0582 \\ Universidade Estadual do Norte do Paraná, Brasil \\ E-mail: camiladalcol@uenp.edu.br \\ Fábio Rodrigues Ferreira Seiva \\ ORCID: https://orcid.org/0000-0002-7461-8773 \\ Universidade Estadual do Norte do Paraná, Brasil \\ E-mail: fabio.seiva@uenp.edu.br \\ Leia Regina de Souza Alcantara \\ ORCID: https://orcid.org/0000-0001-7847-4810 \\ Universidade Estadual do Norte do Paraná, Brasil \\ E-mail: alcantara@uenp.edu.br \\ Carolina Fordellone Rosa Cruz \\ ORCID: https://orcid.org/0000-0002-8936-9191 \\ Universidade Estadual do Norte do Paraná, Brasil \\ E-mail: fordellone@uenp.edu.br \\ Emiliana Cristina Melo \\ ORCID: https://orcid.org/0000-0003-1013-4574 \\ Universidade Estadual do Norte do Paraná, Brasil \\ E-mail: ecmelo@uenp.edu.br
}

\begin{abstract}
Resumo
Este protocolo tem como objetivo mapear as publicações relacionadas ao diagnóstico e tratamento da COVID-19. É um protocolo de revisão de escopo que segue o método preconizado pelo Joanna Briggs Institute, demais autores e a Diretriz Itens de Relatório Preferidos Para Revisões Sistemáticas e Extensão de meta-análises para revisões de escopo. A estratégia PCC (população, conceito e contexto) sistematizou a busca que foi realizada nos meses de maio e junho de 2020, nas bases de dados PubMed, Cochrane, Embase, CINAHL, LILACS, SCOPUS e Web of Science. Quanto à literatura cinzenta, serão usados o Google Scholar. Os resultados serão analisados quantitativamente e serão organizados por temas e apresentados em termos de frequência absoluta e relativa. Espera-se que esta revisão de escopo contribua para o desenvolvimento de estratégias de enfrentamento da doença.
\end{abstract}

Palavras-chave: Diagnóstico; Tratamento farmacológico; Efeito do uso de drogas; Infecções por coronavírus; COVID19; SARS-COV-2.

\begin{abstract}
This protocol is aimed at mapping publications related to diagnosis and treatment of COVID-19. It is a scoping review protocol that follows the method preconized by the Joanna Briggs Institute, other authors and the Guideline Preferred Reporting Items for Systematic reviews and Meta-Analyses extension for Scoping Reviews. The PCC strategy (population, concept and context) did systematize the search that did be performed in the months of May and June of 2020, within PubMed, Cochrane, Embase, CINAHL, LILACS, SCOPUS and Web of Science databases. As for grey literature, Google Scholar will be used. The results will be analyzed quantitatively and will be organized by themes and presented in terms of absolute and relative frequency. It is expected that this scoping review will contribute to the development of strategies for coping with the disease.
\end{abstract}

Keywords: Diagnosis; Pharmacological treatment; Management of drug therapy; Coronavirus infections; COVID-19; SARS-COV-2. 


\section{Resumen}

Este protocolo tiene como objetivo localizar publicaciones relacionadas con el diagnóstico y el tratamiento del COVID19. Es un protocolo scoping review de acuerdo con el método del Instituto Joanna Briggs Institute, otros autores y la guia Elementos de informes preferidos para revisiones sistemáticas y extensión de metaanálisis para revisiones de alcance. La estrategia PCC (población - concepto - contexto) sistematizado la búsqueda que se sucedio a cabo entre mayo y agosto del 2020, en las bases de datos de PubMed, Cochrane, Embase, CINAHL, LILACS, SCOPUS y Web of Science. De igual forma en literatura no convencional: Google Scholar. Os resultados serão analisados quantitativamente e serão organizados por temas e apresentados em termos de frequência absoluta e relativa. Se espera que esta revisión sistemática exploratoria contribuya a elaborar estrategias para enfrentar la enfermedad, así como al desarrollo de políticas de salud pública.

Palabras clave: Diagnóstico; Tratamiento farmacológico; Efecto del uso de drogas; Infecciones por coronavirus; COVID-19; SARS-COV-2.

\section{Introduçãa}

Atualmente, o mundo vem sofrendo um grave problema de saúde pública causado pelo novo coronavírus (SARS-CoV2), o qual atinge uma heterogeneidade de países, pessoas e contextos sociais (Palacios Cruz et al., 2020; Rothan \& Autoimmunity; 2020).

A partir da declaração de emergência em Saúde Pública de Interesse Internacional, oficializada pela Organização Mundial de Saúde (OMS) em 30 de janeiro de 2020, uma sequência de eventos foram recorrentes nos noticiários e nos meios de comunicação científica internacional, como a descrição do nome da doença causada pelo SARS-CoV-2, nominado pela OMS como COVID-19 em 11 de fevereiro do mesmo ano, e no mês seguinte a declaração de pandemia. Até a data de 19 de março de 2021, foram registrados no mundo, 121.464.666 casos confirmados, incluindo 2.684.093 mortes, em 223 países (Coronavirus Disease - COVID-19, [s.d.]; WHO, 2021).

A principal forma relatada de contaminação da COVID-19 é pelo trato respiratório. O contágio ocorre por contato direto com gotículas de saliva e secreções respiratórias, semelhante às formas de contágio descritas em epidemias anteriores de coronavírus (CoVs), como a síndrome respiratória aguda grave (SARS)-CoV em 2003 e a síndrome respiratória do Oriente Médio (MERS)-CoV em 2012 (Li et al., 2020; Botero-Rodríguez, Franco \& Gómez-Restrepo, 2020). Adicionalmente, foi relatado que o SARS-CoV-2 foi isolado de swabs fecais de um paciente com pneumonia, indicando a possibilidade de transmissão por múltiplas vias (Zhang et al., 2020).

De acordo com a etiologia descrita até o momento, o período de incubação do vírus varia de 1 a 14 dias. A COVID-19 é contagiosa durante o período de latência e altamente transmissível em seres humanos de todas as faixas etárias. No entanto, existem grupos populacionais com maior risco para o desenvolvimento de manifestações clínicas e mortalidade, em especial os idosos com idade superior a 60 anos e indivíduos com comorbidades associadas (De Rose et al., 2020).

Em relação ao diagnóstico, o padrão ouro para a detecção do SARS-CoV-2 é baseado em técnicas de biologia molecular, com a identificação do RNA viral, por meio de RT- PCR (Real Time Reverse-Polymerase Chain Reaction) em amostras de $s w a b$ nasal, de garganta ou outras amostras do trato respiratório (Lu; Stratton \& Tang, 2020). Entretanto, em razão da escassez de testes de PCR e de obstáculos para sua operacionalização, o diagnóstico clínico e epidemiológico tem sido adotado a fim de garantir o diagnóstico precoce bem como o controle das manifestações clínicas (Abd El-Aziz \& Stockand, 2020).

A sintomatologia da COVID-19 varia de casos leves a graves, e as manifestações mais comuns incluem febre, tosse, fadiga e falta de ar. Manifestações menos comuns como a cefaleia, diarreia, produção de expectoração, fraqueza muscular, dor de garganta e dor pleurítica também são citadas (Samudrala et al., 2020; Hosseini et al., 2020). Os resultados de pesquisas demonstram que a maioria dos adultos com infecção por SARS-CoV-2 apresentam sintomas leves como febre, mal-estar e tosse, e não necessitam de hospitalização (Huang et al., 2020). Por outro lado, 20\% dos casos requerem hospitalização e destes, 5\% necessitam de cuidados em uma Unidade de Terapia Intensiva, sendo que para cerca de 2,5\% destes casos é necessário suporte 
com ventilação mecânica ou oxigenação por membrana extracorpórea. Pequena parcela de infectados em estado crítico $(0,9 \%$ a 4,4\%) desenvolvem complicações, entre elas a síndrome respiratória aguda grave, insuficiência respiratória, falência de múltiplos órgãos e óbito (Jin et al., 2020).

Até o momento não há medicamentos antivirais específicos para o tratamento da COVID-19 (Holshue et al., 2020; Botero-Rodríguez, Franco \& Gómez-Restrepo, 2020). Entretanto, diversos ensaios clínicos para o tratamento desta infecção viral estão em andamento, e a maioria se refere aos mecanismos moleculares e a organização genômica de SARS-CoV-2. Um avanço científico evidente foi o desenvolvimento de vacinas contra a COVID-19, sendo que algumas demonstraram cerca de 95\% de eficácia clínica e foram aprovadas em diferentes países, entre elas estão a Oxford/AstraZeneca (AZD1222), Moderna Inc (Mrna1273) Sinovac Biotech (Coronavac), Shenzhen Medical Institute (LV-SMENP-DC) e (Covid-19/aAPC) (Chilamakuri \& Agarwal, 2021).

Além destas, a Organização Mundial da Saúde aprovou até a data de 16 de fevereiro de 2021, as vacinas Pfizer/Biotech (BNT122b2/COMIRNATY Tonzinbameram - INN), Serum Institute of Indica (Covishield - ChAdOx1_nCoV19), Sinopharm / BIBP (SARS-CoV-2 Vaccine "Vero Cell" Inactivated - lnCoV), Sinovac (SARS-CoV-2 Vaccine "Vero Cell" - Inactivated), Janssem Johnson \& Johnson (Ad26.COV2.S), CanSionoBio (Ad5-nCoV) (WHO, [s.d.]).

Em relação ao manejo clínico, este se baseia no suporte sintomático como oxigenoterapia, e, em medidas para prevenir infecções e complicações (Abd El-Aziz \& Stockand, 2020). Em paralelo, os esforços coletivos estão centrados em acelerar o desenvolvimento da terapêutica da COVID-19 através da reutilização de drogas que já são clinicamente disponíveis ou de reaproveitamento de drogas antivirais aprovadas pela Food and Drug Administration (Saha et al., 2020; Saul \& Einav, 2020). O uso de drogas antivirais já existentes tem o intuito de enfraquecer a fusão do vírus com a célula hospedeira, bem como minimizar sua multiplicação. Embora a antibioticoterapia não seja padrão para tratamento de pessoas com COVID-19, ela pode ser associada no caso de infecções secundárias (Abd El-Aziz \& Stockand, 2020).

Tendo em vista esta emergência de Saúde Pública e seu impacto no cotidiano das pessoas em todo o mundo, a comunidade científica respondeu rapidamente com investigações sobre a COVID-19 e diversas evidências científicas foram publicadas. Com este olhar, o objetivo deste protocolo de scoping review é mapear as publicações relacionadas a COVID-19, bem como identificar as principais evidências científicas sobre seu diagnóstico e tratamento. Pretende-se a sintetização do conhecimento até então produzido acerca desta temática, o que certamente auxiliará nas estratégias de enfrentamento da doença bem como no desenvolvimento de políticas públicas de saúde, visando a otimização do diagnóstico e tratamento da COVID-19.

\section{Método e Protocolo}

O objetivo do scoping review é identificar e mapear conceitos-chave, tipos de evidências e lacunas relacionadas a uma área definida, pesquisando, selecionando e sintetizando o conhecimento já existente. Neste sentido, será realizado um scoping review referente às publicações associadas ao diagnóstico e tratamento da COVID-19. Para tanto, será aplicada a metodologia proposta por Joanna Briggs Institute (Peters et al., 2020), Arksey \& O’Malley (2005); Tricco et al. (2018), Levac; Colquhoun \& O'Brien (2010), adotando cinco etapas de investigação, a saber: (1) identificar a questão de pesquisa; (2) identificar estudos relevantes; (3) seleção de estudos; (4) extração dos dados; e (5) coletar, resumir e relatar os resultados. Este protocolo será norteado pelos guidelines do Preferred Reporting Items for Systematic reviews and Meta-Analyses extension for Scoping Reviews (PRISMA-ScR) (Peters et al., 2020).

\section{Etapa 1: identificar a questão de pesquisa}

As perguntas a serem pesquisadas seguirão as recomendações do PRISMA-ScR, sendo estruturadas utilizando-se a estratégia PCC: P) População a ser investigada (adultos e idosos); C) Conceito (diagnóstico e tratamento); e C) Contexto 
(COVID-19). Assim, a questão inicial foi: O que há descrito, cientificamente, sobre a COVID-19? Especificamente, procurarse-á responder às seguintes perguntas: Quais os tipos de diagnóstico para COVID-19? Quais tratamentos estão sendo utilizados e/ou testados para a COVID-19?

As palavras-chave e expressões de pesquisas a serem utilizadas incluem: "adult", "elderly", "diagnostic", "treatment", "laboratory techniques", "COVID-19" e "SARS-CoV-2". Os operadores booleanos AND e OR serão combinados entre os descritores.

\section{Etapa 2: identificar estudos relevantes}

A fim de se realizar uma ampla busca na literatura especializada, serão utilizadas as bases de dados eletrônicas de ciências da saúde e multidisciplinares PubMed, Cochrane, Embase, Cumulative Index to Nursing and Allied Health Literature (CINAHL), LILACS, SCOPUS e Web of Science. E, para a busca na literatura cinzenta, o Google Scholar.

As buscas serão operacionalizadas a partir dos termos do Medical Subject Headings (MeSH) e/ou Descritores em Ciências da Saúde (DeCS), combinando-os por meio dos operadores booleanos AND e OR, de acordo com as especificações de cada base de dados.

Na busca, os seguintes filtros serão utilizados: literatura com seres humanos, nos idiomas inglês, espanhol ou português, artigos completos publicados entre o período de $1^{\circ}$ de dezembro de 2019 a 15 de maio de 2020 . O Quadro 1 resume a estratégia adotada nesta etapa para as buscas no PubMed.

Quadro 1 - Estratégia de busca realizada na base de dados PubMed.

\begin{tabular}{|c|c|c|c|}
\hline Estratégia & Descritores & Resultados & Data/hora \\
\hline$\# 1$ & 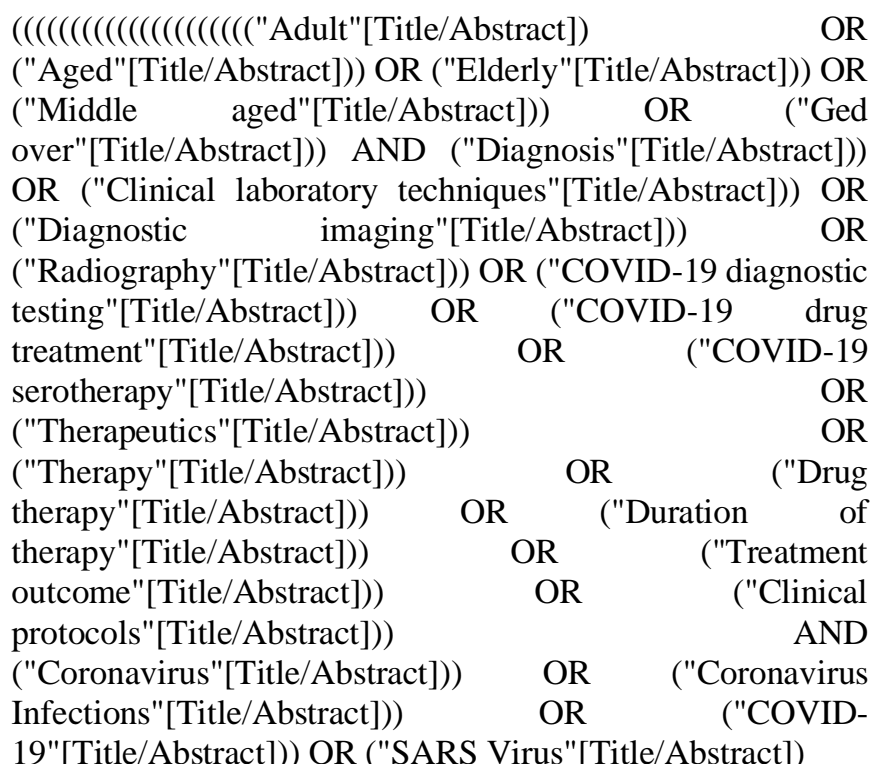 & 2.191 & $\begin{array}{c}14 / 05 / 2020 \\
-23: 36: 25\end{array}$ \\
\hline
\end{tabular}

Fonte: Autores.

\section{Etapa 3: seleção de estudos}

Os estudos incluídos atenderão aos seguintes critérios de elegibilidade: resultados de evidências primárias e secundárias da população adulta acima de 18 anos, que apresentem análises clínicas, laboratoriais ou de imagens, e que sejam detalhadas sobre diagnóstico da COVID-19 e/ou o tratamento da COVID-19, incluindo tratamento medicamentoso e não medicamentoso. 
Serão excluídos os estudos que se referirem à resultados parciais, notas técnicas ou relatórios preliminares, abordagens com foco principal em fisiopatologia, microbiologia e/ou bioquímica da COVID-19, ou estudos sobre o impacto socioeconômico relacionado às estratégias de diagnósticos e tratamentos. Os artigos selecionados e excluídos serão organizados a partir do fluxograma PRISMA-ScR e apresentados conforme a Figura 1.

Figura 1 - fluxograma que ilustra a seleção, exclusão e o total de artigos incluídos e analisados.

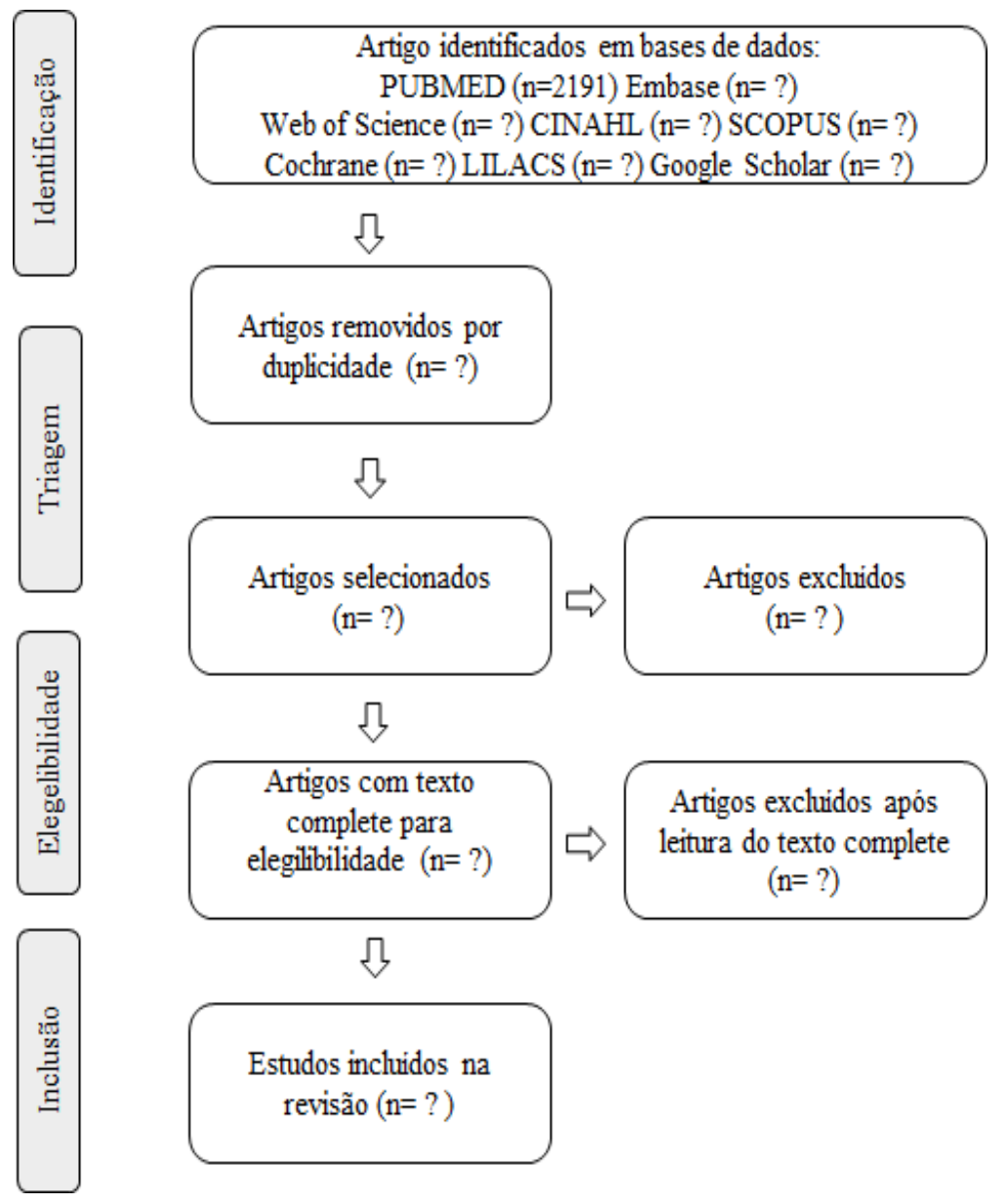

Fonte: Autores de acordo com o PRISMA-ScR.

\section{Etapa 4: extração dos dados}

Nesta etapa serão extraídas informações dos artigos incluídos nesta revisão. Para tanto, os pesquisadores desenvolveram um protocolo para nortear. Os 
Quadro 2 - Itens e subitens para extração dos dados.

\begin{tabular}{|l|l|}
\hline Itens para extração dos dados & Subitens para extração dos dados \\
\hline 1- Características do estudo & Autor, título, ano de publicação, país de origem \\
\hline 2- Desenho do estudo & $\begin{array}{l}\text { Objetivo, tipo de estudo, nível de evidência, tamanho amostral, características } \\
\text { dos participantes }\end{array}$ \\
\hline 3 - Diagnóstico & Tipos de diagnóstico: clínico, laboratorial e/ou imagens \\
\hline 4 - Tipo de tratamento & $\begin{array}{l}\text { Antimalárico, anti-inflamatório, plasma convalescente, anticoagulante, } \\
\text { antibiótico, corticoide, antirretroviral e/ou outros }\end{array}$ \\
\hline 5 - Nome da droga, posologia e eficácia & Cura, óbito, melhora clínica, mudança do tratamento ou ausência de eficácia \\
\hline 6 - Conclusões & Recomendações \\
\hline
\end{tabular}

Fonte: Autores.

Para minimizar eventuais vieses de seleção e análise, os dados serão extraídos por dois pesquisadores separados, de forma independente e, quando houver discordância entre as avaliações, o artigo será submetido para um terceiro pesquisador.

Os estudos identificados serão incluídos no Software State of the Art through Systematic Review (StArt), o qual contribuirá para a sistematização e gerenciamento dos artigos que foram extraídos das bases de dados.

\section{Etapa 5: coletar, resumir e relatar os resultados}

Os dados resultantes deste scoping review serão coletados e resumidos quantitativamente (baseando-se na síntese dos resultados, conclusões e recomendações). Os dados relacionados ao país de origem da publicação serão analisados e georreferenciados por meio do Software ArcGIS versão 10.6. Os demais dados serão analisados por meio de estatística descritiva e inferencial, com apoio do Software SPSS versão 22.0.

Os resultados serão apresentados em temas: caracterização dos estudos incluídos, diagnósticos utilizados para detecção de COVID-19 e tratamentos associados. Esta etapa de análise será conduzida pelos autores e discutida dentro do grupo de revisão para garantir a validade e consistência dos resultados. O formato exato para a apresentação dos resultados dependerá da natureza das evidências a serem exploradas.

\section{Discussão e Considerações Finais}

A pandemia do coronavírus trouxe diversos desafios para os cientistas e pesquisadores, já que em um período curto de tempo o mundo todo se deparou com uma nova doença, clinicamente agressiva. O seu diagnóstico muitas vezes não é imediato, e o tratamento permanece incerto. Esses desafios aumentam ainda mais a transmissão e circulação do vírus que vem se transformando a cada dia e tornando-se cada vez mais letal.

Considerando que a COVID-19 é um problema de saúde pública a nível mundial, atinge altos números de infectados e óbitos, e, até o momento não há evidências científicas sobre o tratamento específico para esta doença, destaca-se a importância da realização deste scoping review.

O diagnóstico precoce da doença é primordial para a detecção, isolamento, tratamento e acompanhamento adequado do paciente infectado pelo coronavírus. O diagnóstico precoce somado ao tratamento correto da COVID-19 poderá impactar no prognóstico do indivíduo, reduzindo as taxas de hospitalização, complicações e mortalidade.

Há uma avalanche de publicações sobre o coronavírus, as quais envolvem a transmissibilidade, formas de contágio, possíveis tratamentos e diagnósticos precoces. Muitos estudos possuem nível de evidência altíssimo e são considerados no meio 
acadêmico como promissores. Outros, apresentam baixa evidência científica e requerem novos estudos ou aprofundamento. Neste cenário de inúmeras publicações sobre o tema, é imprescindível realizar o mapeamento das publicações e estratificá-las pelo nível de evidência, a fim de nortear novas pesquisas que sirvam de embasamento científico para aprimorar o diagnóstico e tratamento da COVID-19. Além disso, este estudo pode inspirar estudos futuros sobre outros aspectos da Covid-19, como prevenção, contenção, vacinação e complicações.

Por se tratar de uma doença recente, ainda desconhecida em sua totalidade, as formas de diagnóstico e tratamento da doença podem mudar ou evoluir ao decorrer do tempo, exigindo uma atualização constante das publicações, as quais fundamentam a tomada de decisão dos profissionais de saúde e dos órgãos de administração pública.

\section{Referências}

Abd El-Aziz, T. M. \& Stockand, J. D. (2020). Recent progress and challenges in drug development against COVID-19 coronavirus (SARS-CoV-2) - an update on the status. Infection, Genetics and Evolution, 83, 2-10. https://doi.org/10.1016/j.meegid.2020.104327

Arksey, H. \& O’Malley, L. (2005). Scoping studies: Towards a methodological framework. International Journal of Social Research Methodology: Theory and Practice, [S. l.], 8(1), 19-32. https://doi.org/10.1080/1364557032000119616

Botero-Rodríguez, F, Franco, O. H \& Gómez-Restrepo, C. (2020). Glosario para una pandemia: el ABC de los conceptos sobre el coronavirus. Biomédica. 40 (2): 12-26. https://doi.org/10.7705/biomedica.5605

Chilamakuri, R. \& Agarwal, S. (2021). COVID-19: Characteristics and Therapeutics, Cells, 10(2):206. https://doi.org/10.3390/cells10020206

Coronavirus disease (COVID-19). https://www.who.int/emergencies/diseases/novel-coronavirus-2019

Palacios Cruz, M., Santos, E., Velázquez Cervantes, M. A. \& León Juárez, M. (2020). COVID-19, una emergencia de salud pública mundial. Revista Clínica Española, 220:0-0. https://doi.org/10.1016/j.rce.2020.03.001

De Rose, D. U., Piersigilli, F., Ronchetti, M. P, Santisi, A., Bersani, I., Dotta, A., Danhaive, O. \& Auriti, C. (2020). Novel Coronavirus disease (COVID-19) in newborns and infants: What we know so far. Italian Journal of Pediatrics, 45(56): 1-8. https://doi.org/10.1186/s13052-020-0820-X

Holshue, M. L. et al. (2020). First Case of 2019 Novel Coronavirus in the United States. The New England Journal of Medicine, 382(10): 929-936. https://doi.org/10.1056/nejmoa2001191

Huang, C., Wang, Y., Li, X., Ren, L., Zhao, J., Hu, Y. \& Lancet, L. Z (2020). Undefined. Clinical features of patients infected with 2019 novel coronavirus in Wuhan, China. Lancet, 295(10223): 497-506. https://doi.org/10.1016/S0140-6736(20)30183-5

JIN, Y. H. et al. (2020). A rapid advice guideline for the diagnosis and treatment of 2019 novel coronavirus (2019-nCoV) infected pneumonia (standard version). Military Medical Research BioMed Central., 7(4): 1-23. https://doi.org/10.1186/s40779-020-0233-6

Levac, D., Colquhoun, H. \& O’brien, K. K. (2010) Scoping studies: Advancing the methodology. Implementation Science, 5(1): 1-9. https://doi.org/10.1186/1748-5908-5-69

LI, Q. et al. (2020). Early Transmission Dynamics in Wuhan, China, of Novel Coronavirus-Infected Pneumonia. New England Journal of Medicine, 382 (13): 1199-1207. https://doi.org/10.1056/NEJMoa2001316

Lu, H., Stratton, C. W. \& Tang, Y. (2020). Outbreak of pneumonia of unknown etiology in Wuhan, China: The mystery and the miracle. Journal Medical Virology, 92(4): 401-02. https://doi.org/10.1002/jmv.25678

Peters, M. D. J., Marnie, C., Tricco, A. C., Pollock, D., Munn, Z., Alexander, L., Mcinerney, P., Godfrey, C. M. \& Khalil, H. (2020). Updated methodological guidance for the conduct of scoping reviews. JBI evidence synthesis, 18 (10): 2119-2126. https://doi.org/10.11124/JBIES-20-00167

Rothan, H. A. \& Byrareddy, S. N (2020). The epidemiology and pathogenesis of coronavirus disease (COVID-19) outbreak. Elsevier, 109: 1-4. https://doi.org/10.1016/j.jaut.2020.102433

Saha, R. P., Sharma, A. R., Singh, M. K., Samanta, S., Bhakta, S., Mandal, S., Bhattacharya, M., Lee S. S. \& Chakraborty, C. (2020). Repurposing drugs, ongoing vaccine, and new therapeutic development initiatives against COVID-19. Pharmacol. 11(1258): 1-33. https://doi.org/10.3389/fphar.2020.01258

Samudrala, P. K., Kumar, P., Choudhary, K., Thakur, N., Wadekar, G. S., Dayaramani, R., Agrawal, M. \& Alexander, A. (2020). Virology, pathogenesis, diagnosis and in-line treatment of COVID-19. European.. Journal of Pharmacology, 883: 1-12. https://doi.org/10.1016/j.ejphar.2020.173375

Saul, S. \& Einav, S.(2020). Old Drugs for a New Virus: Repurposed Approaches for Combating COVID-19. ACS Infect. Dis, 6: 2304-2318. https://doi.org/10.1021/acsinfecdis.0c00343

Hosseini, E. S., Kashani, N. R., Nikzad, H., Azadbakht, J., Bafrani, H. H. \& Kashani, H. H. (2020). The novel coronavirus Disease-2019 (COVID-19): Mechanism of action, detection and recent therapeutic strategies. Virology, 551:1-9. https://doi.org/10.1016/j.virol.2020.08.011

Tricco, A. C. et al. (2018). PRISMA extension for scoping reviews (PRISMA-ScR): Checklist and explanation. Annals of Internal Medicine, 169\&7): 467-473. https://doi.org/10.7326/M18-0850 
Research, Society and Development, v. 10, n. 4, e26010414068, 2021

(CC BY 4.0) | ISSN 2525-3409 | DOI: http://dx.doi.org/10.33448/rsd-v10i4.14068

WHO - World Health Organization. Coronavirus Disease (Covid-19): Vaccines. Status Of Covid-19 Vaccines Within Who Eul/Pq Evaluation Process. https://extranet.who.int/pqweb/sites/default/files/documents/Status_COVID_VAX_16Feb2021.pdf

Zhang, W., Du, R., Li, B., Zheng, X., Yang, X., Hu, B., Wang, Y., Xiao, G., Yam, B., Shi, Z. \& Zhou, P. (2020). Molecular and serological investigation of 2019-nCoV infected patients: implication of multiple shedding routes. Emerg Microbes Infect, 9(1): 386-389. https://doi.org/10.1080/22221751.2020.1729071 